\title{
Mathematical analysis to an adaptive network of the Plasmodium system
}

\author{
Tomoyuki MiYAJI and Isamu OHNISHI
}

(Received February 7, 2006; Revised July 12, 2006)

\begin{abstract}
Adaptive networks are emerging a lot of fields of science, recently. In this paper, we consider about a mathematical model for adaptive network made by the plasmodium. The organism contains a tube network by means of which nutrients and signals circulate through the body. The tube network changes its shape to connect two exits through the shortest path when the organism is put in a maze and food is placed at two exits. Recently, a mathematical model for this adaptation process of the plasmodium has been proposed. Here we analyze it mathematically rigorously. In ring-shaped network and Wheatstone bridge-shaped network, we mainly show that the globally asymptotically stable equilibrium point of the model corresponds to the shortest path connecting two special points on the network in the case where the shortest path is determined uniquely. From the viewpoint of mathematical technique, especially in the case of Wheatstone bridge-shaped network, we show that there is a simple but novel device used here by which we prove the global asymptotic stability, even when Lyapunov function cannot be constructed.
\end{abstract}

Key words: adaptive network, plasmodium, global asymptotic stability, Lyapunov functions.

\section{Introduction}

The plasmodium of true slime mold Physarum polycephalum is a large amoeba-like organism. Its body contains a tube network by means of which nutrients and signals circulate through the body in effective manner.

When food sources were presented to a starved plasmodium that was spread over the entire agar surface, it concentrated at every food source, respectively. Almost the entire plasmodium accumulated at the food sources and covered each of them in order to absorb nutrients[4]. Only a few tube remained connecting the quasi-separated components of the plasmodium through the short path. Nakagaki et al. showed that this simple organism had the ability to find the minimum-length solution of a maze[5, 6]. The connecting tube traces the shortest path even in a complicated maze. Hydrodynamics theory implies that thick short tubes are in principle the

2000 Mathematics Subject Classification : 34D05, 34D23, 37N25. 
most effective for transportation. And this adaptation process of the tube network is based on an underlying physiological mechanism, that is, a tube becomes thicker as a flux in the tube is larger. This insight might be based on the research on the rhythmic oscillation of Physarum polycephalum[7]. Tero et al. made a mathematical model in consideration of the qualitative mechanisms clarified by experiments[8]. According to numerical simulation results, the minimum-length solution of a maze can be obtained as an asymptotic steady state of the ODEs model[ $[8,9]$. To justify this property mathematically rigorously, it is necessary to prove that the globally asymptotically stable equilibrium point corresponds to the shortest path connecting two exits of a maze and the system has neither the oscillating nor chaotic solution. We have done it in some cases in this paper.

In this paper, we analyze the mathematical model for this adaptation process. Especially, we treat some special cases on a simple network mathematically. In $\S 2$, we briefly introduce the mathematical model proposed by Tero et al. [8]. The model consists of an adaptation equation and a network Poisson equation. According to simulation results in [8], if the adaptation equation depends on the flux linearly, the shortest path is always selected. So Tero et al. called this special system Physarum solver.

In $\S 3$, we analyze Physarum solver for some simple networks mathematically. We treat ring-shaped networks and the Wheatstone bridgeshaped network. The ring-shaped network has two paths connecting two food sources, and there are several nodes on two paths. First, we treat the simplest ring-shaped network, and then we generalize it. We prove that the corresponding Physarum solver can find the shortest path connecting two points on the network, where two points correspond to the food sources. As it is not easy to treat a general network, we analyze the case on these simple networks as the foothold first.

The main point is to prove global asymptotic stability of the equilibrium point. It is one of the general proof methods to find a global Lyapunov function $[2,3]$. However, there is no general way to find it. In fact, we do not find any Lyapunov function in the Wheatstone bridge-shaped network case also in this paper. But we have proved the globally asymptotical stability of the equilibrium point corresponding to the shortest path.

In the proof, we reduce the system into a lower dimensional bounded invariant subset and calculate some Lyapunov-like functions at that subset. We can compare values of these functions with each other along some paths 
locally. Moreover, globally asymptotical behavior of the solution is determined by these estimates thanks to confinement of any trajectory in the bounded invariant subset. In this paper, it plays an important role to consider about the problem from the viewpoint of dynamical system. This idea might work in the case of Physarum solver for a general network, although we cannot carry out it completely so far.

Recently, concerning to analysis of global asymptotic stability, M. Fan and X. Zou have established a generic sufficient criteria to construct a Lyapunov function for some class of systems [1]. As it is a different kind of problem, it is difficult to apply to our problem, although it may be an efficient criteria about some problems in a certain area.

\section{Mathematical model}

The mathematical model for the adaptive dynamics of tube networks has been presented by Tero, Kobayashi and Nakagaki in [8]. In this section, we briefly introduce it.

Now we consider the conformation of tubular network of organism as a weighted graph. Each edge of the graph corresponds to a tube, and each node is either a junction of multiple edges or a free end of an edge. The weight corresponds to the length of each tube. Two special nodes corresponding to food sources are named $N_{1}$ and $N_{2}$ and other nodes are $N_{3}, N_{4}, N_{5}, \ldots$ It is assumed that one of the food source nodes $\left(N_{1}\right)$ always works as a source of sol flow, and the other $\left(N_{2}\right)$ works as a sink. The edge between $N_{i}$ and $N_{j}$ is named $M_{i j}$.

The flux through $M_{i j}$ from $N_{i}$ to $N_{j}$ is expressed by the variable $Q_{i j}$. It is assumed that the flow along the tube is approximately Poiseuille flow. Let $L_{i j}$ and $a_{i j}$ be length and radius of the tube corresponding to the edge $M_{i j}$ respectively. And let $\kappa$ be a viscosity coefficient of the sol and $p_{i}$ be a pressure at the node $N_{i}$. Then the flux $Q_{i j}$ is expressed by the formula

$$
Q_{i j}=\frac{\pi a_{i j}^{4}}{8 \kappa} \frac{p_{i}-p_{j}}{L_{i j}}
$$

Let $D_{i j}=\pi a_{i j}^{4} /(8 \kappa) . \quad D_{i j}$ is called a conductivity of the edge $M_{i j}$. The equation above is rewritten as

$$
Q_{i j}=\frac{D_{i j}}{L_{i j}}\left(p_{i}-p_{j}\right)
$$


Notice that $D_{i j}=D_{j i}, L_{i j}=L_{j i}$ and $Q_{i j}=-Q_{j i}$. It is assumed that no capacity at each node. By considering the conservation law of sol we have

$$
\sum_{i} Q_{i j}= \begin{cases}-I_{0} & \text { if } j=1 \\ I_{0} & \text { if } j=2, \\ 0 & \text { otherwise }\end{cases}
$$

where $I_{0}$ is a flux flowing out from the source node (or sinking into the sink node). In this model, $I_{0}$ is given as a constant.

Following evolution equation for $D_{i j}$ was proposed by Tero et al.[8]:

$$
\frac{d}{d t} D_{i j}=r\left(\bar{D} f\left(\frac{\left|Q_{i j}\right|}{\bar{Q}}\right)-D_{i j}\right)
$$

where $r$ is the adaptation rate. Here we consider the characteristic conductivity $\bar{D}$. The characteristic flux $\bar{Q}$ is taken as a flux which keeps the conductivity $\bar{D}$ constant. The function $f(\xi)$ gives a relation between flux and conductivity, which is defined for non-negative $\xi$, is monotone increasing and satisfies $f(0)=0$ and $f(1)=1$. In this paper, we consider $f(\xi)=$ $\xi^{\mu}$, especially $\mu=1$. By setting $t=t^{\prime} / r$ and $D_{i j}=\bar{D} D_{i j}^{\prime}$ and $Q_{i j}=\bar{Q} Q_{i j}^{\prime}$ and omitting ', we have

$$
\frac{d}{d t} D_{i j}=f\left(\left|Q_{i j}\right|\right)-D_{i j}
$$

which is called an adaptation equation. Note that the path length $L_{i j}$ 's are kept constant throughout the process. By appropriately non-dimensionalizing $L_{i j}$ 's and $p_{i}$ 's, the network Poisson equation for the pressure is derived from (2.1) and (2.2) as follows:

$$
\sum_{i} \frac{D_{i j}}{L_{i j}}\left(p_{i}-p_{j}\right)= \begin{cases}-I_{0} & \text { if } j=1 \\ I_{0} & \text { if } j=2 \\ 0 & \text { otherwise. }\end{cases}
$$

By setting $p_{2}=0$ as a basic pressure level, all $p_{i}$ 's can be determined by solving system (2.4).

The evolution of the variables $D_{i j}$ is controled by the adaptation equation (2.3), and $p_{i}$ 's and $Q_{i j}$ 's are determined by solving the network Poisson equation (2.4) characterized by $D_{i j}$ 's at each moment. Again, $Q_{i j}$ affects the evolution of $D_{i j}$ through the adaptation equation (2.3). 


\section{Mathematical analysis of simple networks}

In this section, we analyze the Plasmodium System for some simple networks from the viewpoint of the dynamical system. Though it is not easy to analyze a general case, we expect to be able to give some suggestion by analyzing basic structures involved in a general network.

\subsection{Ring-shaped network}

Now we study the ring-shaped network as shown in Fig. 3.1. This network consists of two nodes $N_{1}, N_{2}$ and two edges connecting them. For simplicity, we replace $L_{12}^{i}, Q_{12}^{i}$ and $D_{12}^{i}(i=1,2)$ by $L_{i}, Q_{i}$ and $D_{i}$, respectively. Then the fluxes along each edge are calculated as

$$
Q_{1}=I_{0} \frac{D_{1} / L_{1}}{D_{1} / L_{1}+D_{2} / L_{2}}, \quad Q_{2}=I_{0} \frac{D_{2} / L_{2}}{D_{1} / L_{1}+D_{2} / L_{2}} .
$$

Since $Q_{1}$ and $Q_{2}$ are nonnegative, the adaptation equation (2.3) becomes

$$
\left\{\begin{array}{l}
\frac{d}{d t} D_{1}=\left(I_{0} \frac{D_{1} / L_{1}}{D_{1} / L_{1}+D_{2} / L_{2}}\right)^{\mu}-D_{1}, \\
\frac{d}{d t} D_{2}=\left(I_{0} \frac{D_{2} / L_{2}}{D_{1} / L_{1}+D_{2} / L_{2}}\right)^{\mu}-D_{2},
\end{array}\right.
$$

where $\mu>0$.

This system has three equilibrium points. Two of them are $A_{1}=$ $\left(D_{1}, D_{2}\right)=\left(I_{0}^{\mu}, 0\right)$ and $A_{2}=\left(0, I_{0}^{\mu}\right)$ that correspond to the state that only one of the two path survives and the other vanishes. And another equilibrium point is

$$
B=\left(D_{1}, D_{2}\right)=\left(\left[\frac{I_{0}}{1+\left(L_{1} / L_{2}\right)^{1 / 1-\mu}}\right]^{\mu},\left[\frac{I_{0}}{1+\left(L_{2} / L_{1}\right)^{1 / 1-\mu}}\right]^{\mu}\right)
$$

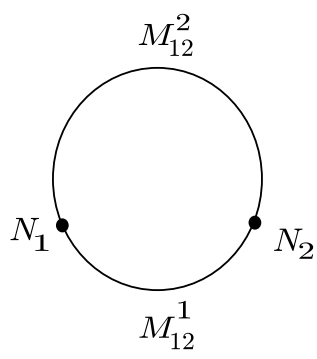

Fig. 3.1. Ring-shaped network. 
for $\mu \neq 1$. $B$ corresponds to the state that both of two edges survive.

The linear stability of these equilibrium points is known by Tero et al. in [8]. If $\mu>1$, then $B$ is unstable, while $A_{1}$ and $A_{2}$ are linear stable. In this case, the final state is determined by the initial state. If $\mu<1$, then $B$ is linear stable, while $A_{1}$ and $A_{2}$ are unstable.

If $\mu=1$, then the equilibrium point $B$ doesn't exist. In this case, the stability of each equilibrium point is determined by the relation between $L_{1}$ and $L_{2}$. That is, if $L_{1}<L_{2}$, then $A_{1}$ is stable and $A_{2}$ is unstable. If $L_{1}>L_{2}$, then $A_{1}$ is unstable, while $A_{2}$ is stable. If $L_{1}=L_{2}$, then every orbit is attracted to the line segment $A_{1} A_{2}$ and the ratio between $D_{1}$ and $D_{2}$ is kept constant along each orbit.

We can find a Lyapunov function for this system as follows:

$$
V\left(D_{1}, D_{2}\right)=\frac{I_{0}^{\mu} D_{1}^{\mu}}{\mu L_{1}^{\mu+1}}+\frac{I_{0}^{\mu} D_{2}^{\mu}}{\mu L_{2}^{\mu+1}}-\frac{1}{\mu+1}\left(\frac{D_{1}}{L_{1}}+\frac{D_{2}}{L_{2}}\right)^{\mu+1} .
$$

The partial derivatives of $V$ are

$$
\begin{aligned}
& \frac{\partial V}{\partial D_{1}}=\frac{I_{0}^{\mu}}{L_{1}^{\mu+1}} D_{1}^{\mu-1}-\frac{1}{L_{1}}\left(\frac{D_{1}}{L_{1}}+\frac{D_{2}}{L_{2}}\right)^{\mu}, \\
& \frac{\partial V}{\partial D_{2}}=\frac{I_{0}^{\mu}}{L_{2}^{\mu+1}} D_{2}^{\mu-1}-\frac{1}{L_{2}}\left(\frac{D_{1}}{L_{1}}+\frac{D_{2}}{L_{2}}\right)^{\mu} .
\end{aligned}
$$

For simplicity, let $S=D_{1} / L_{1}+D_{2} / L_{2}$. The differentiation of the function $V$ along the solution of (3.2) starting from the first quadrant is calculated as follows:

$$
\begin{aligned}
\frac{d}{d t} V\left(D_{1}, D_{2}\right)= & \frac{\partial V}{\partial D_{1}} \frac{d D_{1}}{d t}+\frac{\partial V}{\partial D_{2}} \frac{d D_{2}}{d t} \\
= & \frac{\partial V}{\partial D_{1}}\left\{\left(I_{0} \frac{D_{1} / L_{1}}{S}\right)^{\mu}-D_{1}\right\} \\
& \quad+\frac{\partial V}{\partial D_{2}}\left\{\left(I_{0} \frac{D_{2} / L_{2}}{S}\right)^{\mu}-D_{2}\right\} \\
= & \frac{L_{1} D_{1}}{S^{\mu}}\left[\frac { \partial V } { \partial D _ { 1 } } \left\{\frac{I_{0}^{\mu}}{\left.\left.L_{1}^{\mu+1} D_{1}^{\mu-1}-\frac{S^{\mu}}{L_{1}}\right\}\right]}\right.\right. \\
& \quad+\frac{L_{2} D_{2}}{S^{\mu}}\left[\frac{\partial V}{\partial D_{2}}\left\{\frac{I_{0}^{\mu}}{L_{2}^{\mu+1}} D_{2}^{\mu-1}-\frac{S^{\mu}}{L_{2}}\right\}\right]
\end{aligned}
$$




$$
\begin{aligned}
& =\frac{L_{1} D_{1}}{L_{2} S^{\mu}}\left(\frac{\partial V}{\partial D_{1}}\right)^{2}+\frac{L_{2} D_{2}}{L_{1} S^{\mu}}\left(\frac{\partial V}{\partial D_{2}}\right)^{2} \\
& \geq 0 \quad\left(\text { in } \mathbf{R}_{+}^{2}\right) .
\end{aligned}
$$

Therefore we can conclude that only the equilibrium point is the steady state of (3.2). Especially, if $\mu=1$, then the equilibrium point corresponding to the state that only the shorter edge survives is globally asymptotically stable. This property is appropriate for our problem and concides with the simulation results shown in [8]. Hereafter, we set $\mu=1$ and use $f(\xi)=\xi$ as an adaptation function.

Remark 1 If $\mu=1$, the function

$$
V\left(D_{1}, D_{2}\right)=L_{1} \log \frac{D_{1}}{L_{1}}-L_{2} \log \frac{D_{2}}{L_{2}}
$$

is also a Lyapunov function of (3.2). Actually, we obtain

$$
\dot{V}=\frac{\dot{D}_{1}}{D_{1} / L_{1}}-\frac{\dot{D}_{2}}{D_{2} / L_{2}}=-L_{1}+L_{2}
$$

Hence, the sign of $\dot{V}$ is determined by the length of each edge.

\subsection{Generalization of the ring-shaped network}

Here we generalize the ring-shaped network. We consider two paths $P^{1}, P^{2}$ connecting two food source nodes. Suppose that there are $m$ nodes $N_{1}^{1}, N_{2}^{1}, \ldots, N_{m}^{1}$ on the path $P^{1}$, and $P^{2}$ has $n$ nodes $N_{1}^{2}, N_{2}^{2}, \ldots, N_{n}^{2}$. Here we assume that $N_{1}^{1}=N_{1}^{2}$ and $N_{m}^{1}=N_{n}^{2}$ are food source nodes. There are at least two nodes on one path. We express the conductivity, the length, and the flux through the edge $M_{i, i+1}^{j}(j=1,2)$ from $N_{i}^{j}$ to $N_{i+1}^{j}$ by $D_{i}^{j}, L_{i}^{j}$, and $Q_{i}^{j}$, respectively. The pressure at $N_{i}^{j}$ is written by $p_{i}^{j}$. From our assumption, we have $p_{1}^{1}=p_{1}^{2}$ and $p_{m}^{1}=p_{n}^{2}=0$.

In this case, the network Poisson equation is expressed as

$$
\left\{\begin{array}{l}
a_{1}\left(p_{1}^{1}-p_{2}^{1}\right)+b_{1}\left(p_{1}^{2}-p_{2}^{2}\right)=I_{0}, \\
a_{i}\left(p_{i}^{1}-p_{i+1}^{1}\right)-a_{i+1}\left(p_{i+1}^{1}-p_{i+2}^{1}\right)=0 \\
\quad(i=1,2, \ldots, m-2), \\
b_{j}\left(p_{j}^{2}-p_{j+1}^{2}\right)-b_{j+1}\left(p_{j+1}^{2}-p_{j+2}^{2}\right)=0 \\
\quad(j=1,2, \ldots, n-2), \\
a_{m-1} p_{m-1}^{1}+b_{n-1} p_{n-1}^{2}=I_{0},
\end{array}\right.
$$


where $a_{i}=D_{i}^{1} / L_{i}^{1}, b_{j}=D_{j}^{2} / L_{j}^{2}$. First, we verify that the node $N_{1}^{1}=N_{1}^{2}$ works as a source of flow and $N_{m}^{1}=N_{n}^{2}$ works as a sink. We write the matrix form of (3.4) as follows. Let $C, x$, and $d$ be a coefficient matrix, unknown column vector, and a column vector, respectively. $C=\left(c_{i j}\right)$ is a square matrix of order $(m+n-3)$, and $x$ and $d$ are expressed as

$$
\begin{aligned}
& x={ }^{t}\left(p_{1}^{1}, \ldots, p_{m-1}^{1}, p_{2}^{2}, \ldots, p_{n-1}^{2}\right) \\
& d={ }^{t}\left(I_{0}, 0, \ldots, 0\right),
\end{aligned}
$$

where ${ }^{t} u$ means a transpose of $u$. (3.4) is equivalent to $C x=d$.

Lemma 3.1 If $m+n-3$ is odd, then $\operatorname{det} C$ is positive. If $m+n-3$ is even, then $\operatorname{det} C$ is negative.

Proof. We calculate $\operatorname{det} C$ by elementary operation of matrices. Now $c_{11}=$ $a_{1}+b_{1}, c_{21}=a_{1}, c_{m 1}=b_{1}$ and the other components of the first column are zero. Add the first row multiplied by $-c_{21} / c_{11}$ to the second row. And add the first row multiplied by $-c_{m 1} / c_{11}$ to the $m$ th row. Then we can eliminate the components of the first column except $c_{11}$, and $\operatorname{det} C$ is equal to the product of $c_{11}$ and $\operatorname{det} C^{\prime}$. Here $\operatorname{det} C^{\prime}$ is a square matrix of order $(m+n-4)$. We rewrite $C$ with $C^{0}$. We write the matrix of order $(m+$ $n-3-k)$ obtained at $k$ th step via such a procedure as $C^{k}=\left(c_{i j}^{k}\right)$. Then $\operatorname{det} C^{0}$ can be calculated as

$$
\operatorname{det} C^{0}=\prod_{k=0}^{m+n-4} c_{11}^{k}
$$

We can show that $c_{11}^{k}$ 's are negative for $k \geq 1$ as follows. Add the first row multiplied by $-c_{21}^{k} / c_{11}^{k}$ to the second row, and add the first row multiplied by $-c_{m-k, 1}^{k} / c_{11}^{k}$ to the $(m-k)$ th row for $k=0, \ldots, m-2$. Then we have

$$
c_{11}^{k}=-\frac{\prod_{i=1}^{k+1} a_{i}+b_{1} \sum_{i=1}^{k+1}\left(\prod_{j=1}^{k+1} a_{j}\right) / a_{i}}{\prod_{i=1}^{k} a_{i}+b_{1} \sum_{i=1}^{k}\left(\prod_{j=1}^{k} a_{j}\right) / a_{i}} \quad(k=1, \ldots, m-2) .
$$

And we also have

$$
\begin{aligned}
c_{m-k, 1}^{k}=c_{1, m-k}^{k}=\frac{b_{1} \prod_{i=1}^{k} a_{i}}{\prod_{i=1}^{k} a_{i}+b_{1} \sum_{i=1}^{k}\left(\prod_{j=1}^{k}\right) / a_{j}} & \\
& (k=1, \ldots, m-2) .
\end{aligned}
$$


By using these, we obtain

$$
\begin{aligned}
c_{11}^{m-1}= & -\left(b_{1}+b_{2}\right)+\frac{-c_{21}^{m-2}}{c_{11}^{m-2}} c_{12}^{m-2} \\
=-\left(b_{1}+b_{2}\right)+ & \frac{\left(b_{1} \prod_{j=1}^{m-2} a_{j}\right)^{2}}{\left(\prod_{j=1}^{m-1} a_{j}+b_{1} \sum_{j=1}^{m-1}\left(\prod_{k=1}^{m-1} a_{k}\right) / a_{j}\right)} \\
& \times \frac{1}{\left(\prod_{j=1}^{m-2} a_{j}+b_{1} \sum_{j=1}^{m-2}\left(\prod_{k=1}^{m-2} a_{k}\right) / a_{j}\right)} \\
\leq-\left(b_{1}+b_{2}\right)+ & b_{1}=-b_{2} \leq 0 .
\end{aligned}
$$

Inductively, we have

$$
c_{11}^{m+k}=-\left(b_{k+2}+b_{k+3}\right)-\frac{\left(b_{k+2}\right)^{2}}{c_{11}^{m+k-1}} \quad(k=0,1, \ldots, n-4) .
$$

Here $c_{11}^{m+k} \leq-b_{k+3}$. In fact, if $k=0$, then

$$
c_{11}^{m}=-\left(b_{2}+b_{3}\right)-\frac{\left(b_{2}\right)^{2}}{c_{11}^{m-1}} \leq-\left(b_{2}+b_{3}\right)+\frac{\left(b_{2}\right)^{2}}{b_{2}}=-b_{3} .
$$

Now we assume that $c_{11}^{m+i} \leq-b_{i+3}$ holds. If $k=i+1$, then

$$
\begin{aligned}
c_{11}^{m+i+1}=-\left(b_{i+3}+b_{i+4}\right)- & \frac{\left(b_{i+3}\right)^{2}}{c_{11}^{m+i}} \\
& \leq-\left(b_{i+3}+b_{i+4}\right)+\frac{\left(b_{i+3}\right)^{2}}{b_{i+3}}=-b_{i+4} .
\end{aligned}
$$

Hence, the inequality holds for every $k$.

Therefore $c_{11}^{k}$ 's are negative for $k \geq 1$. This implies that the sign of $\operatorname{det} C$ is determined by the parity of $(m+n-3)$.

Lemma $3.2 p_{m-1}^{1} \geq 0$ and $p_{n-1}^{2} \geq 0$.

Proof. Let $C_{i}^{0}$ be a matrix which is obtained from $C^{0}$ by replacing the $i$ th column by the column vector $d$. According to Cramer's formula, $p_{m-1}^{1}$ and $p_{n-1}^{2}$ are calculated as

$$
p_{m-1}^{1}=\frac{\operatorname{det} C_{m-1}^{0}}{\operatorname{det} C^{0}}, \quad p_{n-1}^{2}=\frac{\operatorname{det} C_{m+n-3}^{0}}{\operatorname{det} C^{0}} .
$$


And we can caluculate $\operatorname{det} C_{m-1}^{0}$ and $\operatorname{det} C_{m+n-3}^{0}$ as

$$
\begin{aligned}
\operatorname{det} C_{m-1}^{0} & =(-1)^{m+n-4} I_{0}\left(\prod_{i=1}^{m-2} a_{i}\right)\left(\prod_{i=1}^{n-2}\left(b_{i}+b_{i+1}\right)\right), \\
\operatorname{det} C_{m+n-3}^{0} & =(-1)^{m+n-4} I_{0}\left(\prod_{i=1}^{n-2} b_{i}\right) \sum_{i=1}^{m-1}\left(\frac{\prod_{j=1}^{m-1} a_{j}}{a_{i}}\right) .
\end{aligned}
$$

The signs of $\operatorname{det} C_{m-1}^{0}$ and $\operatorname{det} C_{m+n-3}^{0}$ coincide that of $\operatorname{det} C^{0}$. Therefore, we obtain $p_{m-1}^{1} \geq 0$ and $p_{n-1}^{2} \geq 0$.

Lemma 3.3 $Q_{i}^{1} \geq 0 \quad(i=1,2, \ldots, m-1)$ and $Q_{j}^{2} \geq 0 \quad(j=1,2, \ldots$, $n-1)$.

Proof. From the network Poisson equation (3.4), we obtain

$$
\begin{cases}a_{m-1} p_{m-1}^{1}=Q_{i}^{1}=a_{i}\left(p_{i}^{1}-p_{i+1}^{1}\right) & (i=1, \ldots, m-2) \\ b_{n-1} p_{n-1}^{2}=Q_{j}^{2}=b_{j}\left(p_{j}^{2}-p_{j+1}^{2}\right) & (j=1, \ldots, n-2)\end{cases}
$$

Hence, the assertion follows from the previous lemma.

Therefore, the node $N_{1}^{1}=N_{1}^{2}$ works as a source of flow and $N_{m}^{1}=N_{n}^{2}$ works as a sink. The adaptation equation is expressed as

$$
\begin{cases}\frac{d}{d t} D_{i}^{1}=Q_{i}^{1}-D_{i}^{1} & (i=1, \ldots, m-1) \\ \frac{d}{d t} D_{j}^{2}=Q_{j}^{2}-D_{j}^{2} & (j=1, \ldots, n-1) .\end{cases}
$$

We can find a Lyapunov function for this system.

Proposition 3.4 The function

$$
V=\sum_{i=1}^{m-1} L_{i}^{1} \log \frac{D_{i}^{1}}{L_{i}^{1}}-\sum_{j=1}^{n-1} L_{j}^{2} \log \frac{D_{j}^{2}}{L_{j}^{2}}
$$

is a Lyapunov function for the system (3.8).

Proof. Let $V_{i}^{1}=\log \left(D_{i}^{1} / L_{i}^{1}\right)(i=1,2, \ldots, m-1)$, then

$$
\dot{V}_{i}^{1}=\frac{1}{D_{i}^{1} / L_{i}^{1}}\left(Q_{i}^{1}-D_{i}^{1}\right)=\left(p_{i}^{1}-p_{i+1}^{1}\right)-L_{i}^{1} .
$$


Hence, we obtain

$$
\sum_{i=1}^{m-1} \dot{V}_{i}^{1}=p_{1}^{1}-p_{m}^{1}-\sum_{i=1}^{m-1} L_{i}^{1}
$$

Similarly, let $V_{j}^{2}=\log \left(D_{j}^{2} / L_{j}^{2}\right)(j=1,2, \ldots, n-1)$, then $\dot{V}_{j}^{2}=\left(p_{j}^{2}-\right.$ $\left.p_{j+1}^{2}\right)-L_{j}^{2}$. Hence, we obtain

$$
\sum_{j=1}^{n-1} \dot{V}_{j}^{2}=p_{1}^{2}-p_{n}^{2}-\sum_{j=1}^{n-1} L_{j}^{2}
$$

Since $p_{1}^{1}=p_{1}^{2}$ and $p_{m}^{1}=p_{n}^{2}=0$, we have

$$
\dot{V}=\sum_{i=1}^{m-1} \dot{V}_{i}^{1}-\sum_{j=1}^{n-1} \dot{V}_{j}^{2}=-\sum_{i=1}^{m-1} L_{i}^{1}+\sum_{j=1}^{n-1} L_{j}^{2}
$$

Therefore, the sign of $\dot{V}$ is determined by the difference between the lengths of two paths.

Let $W$ be a subset such that

$$
W=\left\{\begin{array}{l|l}
D \in \mathbf{R}_{+}^{m+n-2} & \begin{array}{c}
D_{1}^{1}+D_{1}^{2}=I_{0}, D_{i}^{1}=D_{i+1}^{1}, \\
D_{j}^{2}=D_{j+1}^{2}
\end{array}
\end{array}\right\},
$$

where $D=\left(D_{1}^{1}, \ldots, D_{m-1}^{1}, D_{2}^{2}, \ldots, D_{n-1}^{2}\right)$. We can restrict $(3.8)$ on $W$.

Lemma 3.5 $W$ is an exponentially attracting invariant subset of (3.8).

Proof. It is obvious from the network Poisson equation (3.4). We obtain

$$
\begin{aligned}
& \frac{d}{d t}\left(D_{1}^{1}+D_{1}^{2}\right)=I_{0}-\left(D_{1}^{1}+D_{1}^{2}\right), \\
& \frac{d}{d t}\left(D_{i}^{1}-D_{i+1}^{1}\right)=-\left(D_{i}^{1}-D_{i+1}^{1}\right) \quad(i=1,2, \ldots, m-2), \\
& \frac{d}{d t}\left(D_{j}^{2}-D_{j+1}^{2}\right)=-\left(D_{j}^{2}-D_{j+1}^{2}\right) \quad(j=1,2, \ldots, n-2) .
\end{aligned}
$$

Hence, any positive orbit of (3.8) is attracted to $W$ exponentially.

Therefore, the asymptotic behavior of the solution of (3.8) is determined by the dynamics of $D_{1}^{1}$ on the interval $\left[0, I_{0}\right]$. The Lyapunov function $V$ 
becomes

$$
V=\sum_{i=1}^{m-1} L_{i}^{1} \log \frac{D_{1}^{1}}{L_{i}^{1}}-\sum_{j=1}^{n-1} L_{j}^{2} \log \frac{I_{0}-D_{1}^{1}}{L_{j}^{2}}
$$

on $W$. Hence, if $P_{1}$ is shorter than $P_{2}$, then $\dot{V}>0$ and $D_{1}^{1} \rightarrow I_{0}$ as $t \rightarrow$ $+\infty$. In this case, the equilibrium point of $D_{i}^{1}=I_{0}, D_{j}^{2}=0$ is globally asymptotically stable. On the other, if $P_{2}$ is shorter than $P_{1}$, then $\dot{V}<0$ and $D_{1}^{1} \rightarrow 0$ as $t \rightarrow+\infty$. In this case, the equilibrium point of $D_{i}^{1}=$ $0, D_{j}^{2}=I_{0}$ is globally asymptotically stable.

Theorem 3.6 The system (3.8) can find the shortest path connecting two food source nodes on a general ring-shaped network. That is,

1. If $\sum L_{i}^{1}<\sum L_{j}^{2}$, then the equilibrium point $\left(D_{i}^{1}, D_{j}^{2}\right)=\left(I_{0}, 0\right)$ is globally asymptotically stable.

2. If $\sum L_{i}^{1}>\sum L_{j}^{2}$, then the equilibrium point $\left(D_{i}^{1}, D_{j}^{2}\right)=\left(0, I_{0}\right)$ is globally asymptotically stable.

Here $i=1,2, \ldots, m-1$ and $j=1,2, \ldots, n-1$.

\subsection{Wheatstone bridge-shaped network}

Next, we study the Wheatstone bridge-shaped network as shown in Fig.

3.2. For simplicity, we introduce new variables:

$$
\begin{aligned}
& a=\frac{D_{13}}{L_{13}}, b=\frac{D_{14}}{L_{14}}, c=\frac{D_{32}}{L_{32}}, d=\frac{D_{42}}{L_{42}}, e=\frac{D_{34}}{L_{34}}, \\
& S=a b c+a b d+a c d+a c e+b c d+a d e+b c e+b d e .
\end{aligned}
$$

By the conservation law (2.2) (This is Kirchhoff's law), in this case we possess the following formula:

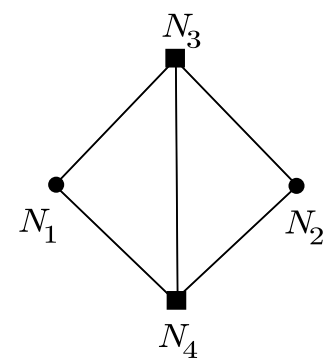

Fig. 3.2. Wheatstone bridge-shaped network. 


$$
\left\{\begin{array}{l}
Q_{13}+Q_{14}=I_{0} \\
Q_{13}+Q_{43}=Q_{32} \\
Q_{14}+Q_{34}=Q_{42} \\
Q_{32}+Q_{42}=I_{0}
\end{array}\right.
$$

Under consideration of (2.1), we therefore get the following network Poisson equation:

$$
\left\{\begin{array}{l}
a\left(p_{1}-p_{3}\right)+b\left(p_{1}-p_{4}\right)=I_{0}, \\
a\left(p_{1}-p_{3}\right)+e\left(p_{4}-p_{3}\right)=c\left(p_{3}-p_{2}\right), \\
b\left(p_{1}-p_{4}\right)+e\left(p_{3}-p_{4}\right)=d\left(p_{4}-p_{2}\right), \\
c\left(p_{3}-p_{2}\right)+d\left(p_{4}-p_{2}\right)=I_{0} .
\end{array}\right.
$$

By setting $p_{2}=0$, we obtain

$$
\begin{aligned}
& p_{1}=\frac{I_{0}}{S}(a b+a d+b c+a e+b e+c d+c e+d e), \\
& p_{3}=\frac{I_{0}}{S}(a b+a d+a e+b e), \quad p_{4}=\frac{I_{0}}{S}(a b+b c+a e+b e) .
\end{aligned}
$$

Hence, $Q_{i j}$ 's are calculated as

$$
\begin{aligned}
Q_{13} & =\frac{a I_{0}}{S}(b c+c d+c e+d e), & Q_{14}=\frac{b I_{0}}{S}(a d+c d+c e+d e), \\
Q_{32} & =\frac{c I_{0}}{S}(a d+a b+a e+b e), & Q_{42}=\frac{d I_{0}}{S}(b c+a b+a e+b e), \\
Q_{34} & =\frac{e I_{0}}{S}(a d-b c) . &
\end{aligned}
$$

Therefore, the adaptation equation is given by

$$
\left\{\begin{array}{l}
\dot{a}=\frac{a I_{0}}{S L_{13}}(b c+c d+c e+d e)-a, \\
\dot{b}=\frac{b I_{0}}{S L_{14}}(a d+c d+c e+d e)-b, \\
\dot{c}=\frac{c I_{0}}{S L_{32}}(a d+a b+a e+b e)-c, \\
\dot{d}=\frac{d I_{0}}{S L_{42}}(b c+a b+a e+b e)-d, \\
\dot{e}=\frac{e I_{0}}{S L_{34}}|a d-b c|-e .
\end{array}\right.
$$

Wheatstone bridge-shaped network is quite different from other exam- 
ples. In the previous examples, the direction of the flow is one-way traffic. However, the direction of the flow along the edge connecting $N_{3}$ and $N_{4}$ can change depending on the initial condition and the length of each edge in this case. Therefore, the term $|a d-b c|$ appears at the right of the equation of $e$. Since such a path can exist also in a general network, we should consider this network.

The system (3.14) has four equilibrium points as paths connecting $N_{1}$ and $N_{2}$. They are

$$
\begin{array}{lll}
A_{1}=\left(\frac{I_{0}}{L_{13}}, 0,0, \frac{I_{0}}{L_{42}}, \frac{I_{0}}{L_{34}}\right), & A_{2}=\left(0, \frac{I_{0}}{L_{14}}, \frac{I_{0}}{L_{32}}, 0, \frac{I_{0}}{L_{34}}\right), \\
A_{3}=\left(\frac{I_{0}}{L_{13}}, 0, \frac{I_{0}}{L_{32}}, 0,0\right), & A_{4}=\left(0, \frac{I_{0}}{L_{14}}, 0, \frac{I_{0}}{L_{42}}, 0\right) .
\end{array}
$$

They correspond to the state that only the path $N_{1} \rightarrow N_{3} \rightarrow N_{4} \rightarrow N_{2}$, $N_{1} \rightarrow N_{4} \rightarrow N_{3} \rightarrow N_{2}, N_{1} \rightarrow N_{3} \rightarrow N_{2}$, or $N_{1} \rightarrow N_{4} \rightarrow N_{2}$ survives, respectively.

Proposition 3.7 Following function is a Lyapunov function of (3.14):

$$
V=\alpha \log a+\beta \log b+\gamma \log c+\delta \log d-L_{34} \log e,
$$

where coefficients $\alpha, \beta, \gamma, \delta$ are determined later in the proof below.

Proof. Let A be an arbitrary positive number.

$$
\begin{aligned}
\dot{V}=\alpha \frac{\dot{a}}{a} & +\beta \frac{\dot{b}}{b}+\gamma \frac{\dot{c}}{c}+\delta \frac{\dot{d}}{d}-L_{34} \frac{\dot{e}}{e} \\
= & \frac{I_{0}}{S}\left\{\left(\frac{\alpha}{L_{13}}+\frac{\beta}{L_{14}}\right)(c d+d e+e c)\right. \\
& +\left(\frac{\gamma}{L_{32}}+\frac{\delta}{L_{42}}\right)(a b+b e+e a) \\
& \left.+\left(\frac{\beta}{L_{14}}+\frac{\gamma}{L_{32}}\right) a d+\left(\frac{\alpha}{L_{13}}+\frac{\delta}{L_{42}}\right) b c-|a d-b c|\right\} \\
& -\left(\alpha+\beta+\gamma+\delta-L_{34}\right) .
\end{aligned}
$$

If we choose coefficients so that the following holds: 


$$
\left\{\begin{array}{l}
\alpha+\beta+\gamma+\delta-L_{34}=A \\
\frac{\alpha}{L_{13}}+\frac{\beta}{L_{14}}=0 \\
\frac{\gamma}{L_{32}}+\frac{\delta}{L_{42}}=0 \\
\frac{\beta}{L_{14}}+\frac{\gamma}{L_{32}}=-1 \quad\left(=-\left(\frac{\alpha}{L_{13}}+\frac{\delta}{L_{42}}\right)\right),
\end{array}\right.
$$

then we have

$$
\dot{V}=\frac{-I_{0}}{S}\{(a d-b c)+|a d-b c|\}-A \leq 0 .
$$

Actually the solution of the linear equations (3.16) is given by

$$
\left\{\begin{array}{l}
\alpha=\frac{L_{13}\left(A+L_{34}+L_{32}-L_{42}\right)}{L_{13}+L_{32}-L_{14}-L_{42}} \\
\beta=\frac{-L_{14}\left(A+L_{34}+L_{32}-L_{42}\right)}{L_{13}+L_{32}-L_{14}-L_{42}} \\
\gamma=\frac{L_{32}\left(A+L_{34}+L_{14}-L_{13}\right)}{L_{13}+L_{32}-L_{14}-L_{42}} \\
\delta=\frac{-L_{42}\left(A+L_{34}+L_{14}-L_{13}\right)}{L_{13}+L_{32}-L_{14}-L_{42}} .
\end{array}\right.
$$

Hence, only if $L_{13}+L_{32} \neq L_{14}+L_{42}$, then $V$ becomes a Lyapunov function, as the positive number $A$ and the coefficients can be suitably taken.

We can reduce (3.14) to three-dimensional system.

Lemma 3.8 Let

$$
W=\left\{(a, b, c, d, e) \in \mathbf{R}_{+}^{5} \mid L_{13} a+L_{14} b=L_{32} c+L_{42} d=I_{0}\right\} .
$$

Then $W$ is an attracting invariant subset of (3.14).

Proof. Now let $P_{1}=L_{13} a+L_{14} b$ and $P_{2}=L_{32} c+L_{42} d$, then we have $\dot{P}_{i}=I_{0}-P_{i}(i=1,2)$. Therefore, any positive orbit of (3.14) is attracted to the three-dimensional invariant subset $W$.

Then we can determine the stability of $A_{1}$ and $A_{2}$.

\section{Lemma 3.9}

1. If the path $N_{1} \rightarrow N_{3} \rightarrow N_{4} \rightarrow N_{2}$ is the shortest, the equilibrium point $A_{1}$ is asymptotically stable. Otherwise, $A_{1}$ is unstable. 
2. If the path $N_{1} \rightarrow N_{4} \rightarrow N_{3} \rightarrow N_{2}$ is the shortest, $A_{2}$ is asymptotically stable. Otherwise, $A_{2}$ is unstable.

Proof. The path $N_{1} \rightarrow N_{3} \rightarrow N_{4} \rightarrow N_{2}$ is the shortest if

$$
L_{13}+L_{34}<L_{14} \quad \text { and } \quad L_{42}+L_{34}<L_{32} .
$$

Eigenvalues of $A_{1}$ are calculated as

$$
-1, \quad \frac{L_{13}+L_{34}-L_{14}}{L_{34}}, \quad \frac{L_{42}+L_{34}-L_{32}}{L_{34}}
$$

with respect to the system (3.14) restricted on $W$. All the eigenvalues become negative if and only if $L_{13}+L_{34}<L_{14}$ and $L_{42}+L_{34}<L_{32}$. That is, $A_{1}$ becomes asymptotically stable if the path $N_{1} \rightarrow N_{3} \rightarrow N_{4} \rightarrow N_{2}$ is the shortest. On the other,the path $N_{1} \rightarrow N_{4} \rightarrow N_{3} \rightarrow N_{2}$ is the shortest if

$$
L_{14}+L_{34}<L_{13} \quad \text { and } \quad L_{32}+L_{34}<L_{42} .
$$

And eigenvalues of $A_{2}$ are calculated as

$$
-1, \quad \frac{L_{14}+L_{34}-L_{13}}{L_{34}}, \quad \frac{L_{32}+L_{34}-L_{42}}{L_{34}} .
$$

All the eigenvalues become negative if and only if $L_{14}+L_{34}<L_{13}$ and $L_{32}+L_{34}<L_{42}$. That is, $A_{2}$ becomes asymptotically stable if the path $N_{1} \rightarrow N_{4} \rightarrow N_{3} \rightarrow N_{2}$ is the shortest.

But the linear stability analysis fails in the other two equilibrium points.

Now we assume $L_{14} b=I_{0}-L_{13} a$ and $L_{42} d=I_{0}-L_{32} c$ and consider $(a, c, e)$-space. Four equilibrium points are represented by

$$
\begin{array}{ll}
A_{1}=\left(\frac{I_{0}}{L_{13}}, 0, \frac{I_{0}}{L_{34}}\right), & A_{2}=\left(0, \frac{I_{0}}{L_{32}}, \frac{I_{0}}{L_{34}}\right), \\
A_{3}=\left(\frac{I_{0}}{L_{13}}, \frac{I_{0}}{L_{32}}, 0\right), & A_{4}=(0,0,0) .
\end{array}
$$

Since all variables are nonnegative and

$$
-e \leq \dot{e} \leq \frac{I_{0}}{L_{34}}-e,
$$

we have only to consider the dynamical system in the hexahedron 


$$
H=\left\{\begin{array}{l|c}
(a, c, e) \in \mathbf{R}_{+}^{3} & \begin{array}{c}
0 \leq a \leq \frac{I_{0}}{L_{13}}, 0 \leq c \leq \frac{I_{0}}{L_{32}}, \\
0 \leq e \leq \frac{I_{0}}{L_{34}}
\end{array}
\end{array}\right\} .
$$

\section{Lemma 3.10}

1. If the path $N_{1} \rightarrow N_{3} \rightarrow N_{4} \rightarrow N_{2}$ is the shortest, the equilibrium point $A_{1}$ is globally asymptotically stable.

2. If the path $N_{1} \rightarrow N_{4} \rightarrow N_{3} \rightarrow N_{2}$ is the shortest, $A_{2}$ is globally asymptotically stable.

Proof. At first, we assume that (3.19) holds. We consider two functions

$$
\begin{aligned}
& V_{1}=L_{13} \log a+L_{34} \log e-L_{14} \log \frac{I_{0}-L_{13} a}{L_{14}}, \\
& V_{2}=L_{42} \log \frac{I_{0}-L_{32} c}{L_{42}}+L_{34} \log e-L_{32} \log c .
\end{aligned}
$$

Their derivatives with respect to time are calculated as

$$
\begin{aligned}
& \dot{V}_{1}=L_{14}-L_{13}-L_{34}+\frac{I_{0}}{S}\{|a d-b c|-(a d-b c)\}>0, \\
& \dot{V}_{2}=L_{32}-L_{42}-L_{34}+\frac{I_{0}}{S}\{|a d-b c|-(a d-b c)\}>0 .
\end{aligned}
$$

As the variables are bounded, $a \rightarrow I_{0} / L_{13}$ and $c \rightarrow 0$ as $t \rightarrow \infty$. Then we obtain $e \rightarrow I_{0} / L_{34}$ as $t \rightarrow \infty$.

Next, we assume that (3.20) holds. We consider two functions

$$
\begin{aligned}
& V_{3}=L_{13} \log a-L_{34} \log e-L_{14} \log \frac{I_{0}-L_{13} a}{L_{14}}, \\
& V_{4}=L_{42} \log \frac{I_{0}-L_{32} c}{L_{42}}-L_{34} \log e-L_{32} \log c .
\end{aligned}
$$

Their derivatives with respect to time are calculated as

$$
\begin{aligned}
& \dot{V}_{3}=-L_{13}+L_{14}+L_{34}-\frac{I_{0}}{S}\{|a d-b c|+(a d-b c)\}<0, \\
& \dot{V}_{4}=-L_{42}+L_{32}+L_{34}-\frac{I_{0}}{S}\{|a d-b c|+(a d-b c)\}<0 .
\end{aligned}
$$

As the variables are bounded, $a \rightarrow 0$ and $c \rightarrow I_{0} / L_{32}$ as $t \rightarrow \infty$. Then we obtain $e \rightarrow I_{0} / L_{34}$ as $t \rightarrow \infty$. 


\section{Lemma 3.11}

1. If the path $N_{1} \rightarrow N_{3} \rightarrow N_{2}$ is the shortest, the equilibrium point $A_{3}$ is globally asymptotically stable.

2. If the path $N_{1} \rightarrow N_{4} \rightarrow N_{2}$ is the shortest, the equilibrium point $A_{4}$ is globally asymptotically stable.

Proof. At first, we show that $A_{3}$ is asymptotically stable if the path $N_{1} \rightarrow$ $N_{3} \rightarrow N_{2}$ is the shortest. In this case, the length of each edge must satisfy

$$
\left\{\begin{aligned}
L_{13}+L_{32}<L_{14}+L_{42} & \text { and } \\
L_{13}<L_{14}+L_{34} & \text { and } \\
L_{32}<L_{42}+L_{34} . &
\end{aligned}\right.
$$

Now we consider the function

$$
V_{L}=L_{14} \log \frac{I_{0}-L_{13} a}{L_{14}}+L_{42} \log \frac{I_{0}-L_{32} c}{L_{42}}-L_{13} \log a-L_{32} \log c .
$$

The derivative of $V_{L}$ with respect to time $t$ is caluculated as

$$
\dot{V}_{L}=L_{13}+L_{32}-L_{14}-L_{42} .
$$

As $L_{13}+L_{32}<L_{14}+L_{42}$, we have $\dot{V}_{L}<0$. Since variables are bounded, $a$ tends to $I_{0} / L_{13}$ or $c$ tends to $I_{0} / L_{32}$ as $t \rightarrow \infty$. First, we assume $a \rightarrow I_{0} / L_{13}$ and consider the dynamics on one of surfaces of $H$,

$$
\left\{(a, c, e) \mid a=\frac{I_{0}}{L_{13}}, 0 \leq c \leq \frac{I_{0}}{L_{32}}, 0 \leq e \leq \frac{I_{0}}{L_{34}}\right\} .
$$

In this case, the dynamics on this surface is equivalent to the triangle-shaped network which consists of three nodes $N_{2}, N_{3}$ and $N_{4}$, where $N_{3}$ works as a source node and $N_{2}$ is a sink. That is, this corresponds to the generalized ring-shaped network of $(m, n)=(3,2)$. As $L_{32}<L_{42}+L_{34}$, the path $N_{3} \rightarrow N_{2}$ survives. Hence, if $a \rightarrow I_{0} / L_{13}$, then $A_{3}$ is globally attracting. On the other, we assume $c \rightarrow I_{0} / L_{32}$ and consider the dynamics on one of surfaces of $H$,

$$
\left\{(a, c, e) \mid c=\frac{I_{0}}{L_{32}}, 0 \leq a \leq \frac{I_{0}}{L_{13}}, 0 \leq e \leq \frac{I_{0}}{L_{34}}\right\} .
$$

In this case, the dynamics on this surface is equivalent to the triangle-shaped network which consists of three nodes $N_{1}, N_{3}$ and $N_{4}$, where $N_{3}$ works as a sink node and $N_{1}$ is a source. As $L_{13}<L_{14}+L_{34}$, the path $N_{1} \rightarrow N_{3}$ 
survives. Hence, if $a \rightarrow I_{0} / L_{13}$, then $A_{3}$ is globally attracting. Therefore, if the path $N_{1} \rightarrow N_{3} \rightarrow N_{2}$ is the shortest, the equilibrium point $A_{3}$ is globally asymptotically stable.

Next, we study $A_{4}$. If the path $N_{1} \rightarrow N_{4} \rightarrow N_{2}$ is the shortest, then

$$
\left\{\begin{aligned}
L_{14}+L_{42}<L_{13}+L_{32} & \text { and } \\
L_{14}<L_{13}+L_{34} & \text { and } \\
L_{42}<L_{32}+L_{34} . &
\end{aligned}\right.
$$

Since $L_{14}+L_{42}<L_{13}+L_{32}$, we have $\dot{V}_{L}>0$. And it follows that $a$ tends to 0 or $c$ tends to 0 as $t \rightarrow \infty$. We assume $a \rightarrow 0$ and consider the dynamics on one of surfaces of $H$,

$$
\left\{(a, c, e) \mid a=0,0 \leq c \leq \frac{I_{0}}{L_{32}}, 0 \leq e \leq \frac{I_{0}}{L_{34}}\right\} .
$$

In this case, the dynamics on this surface is equivalent to the triangle-shaped network which consists of three nodes $N_{2}, N_{3}$ and $N_{4}$, where $N_{4}$ works as a sourse node and $N_{2}$ is a sink. Since $L_{42}<L_{32}+L_{34}$, the path $N_{4} \rightarrow N_{2}$ survives. Hence, if $a \rightarrow 0$, then $A_{4}$ is globally attracting. On the other, we assume $c \rightarrow 0$ and consider the dynamics on one of surfaces of $H$,

$$
\left\{(a, c, e) \mid c=0,0 \leq a \leq \frac{I_{0}}{L_{13}}, 0 \leq e \leq \frac{I_{0}}{L_{34}}\right\} .
$$

In this case, the dynamics on this surface is equivalent to the triangle-shaped network which consists of three nodes $N_{1}, N_{3}$ and $N_{4}$, where $N_{4}$ works as a sink node and $N_{1}$ is a source. As $L_{14}<L_{13}+L_{34}$, the path $N_{1} \rightarrow N_{4}$ survives. Hence, if $c \rightarrow 0$, then $A_{4}$ is globally attracting. Therefore, if the path $N_{1} \rightarrow N_{4} \rightarrow N_{2}$ is the shortest, the equilibrium point $A_{4}$ is globally asymptotically stable.

As a result of the discussion of this section, we obtain the following theorem.

Theorem 3.12 The system (3.14) can find the shortest path connecting $N_{1}$ and $N_{2}$ of the Wheatstone bridge-shaped network. That is, the equilibrium point of (3.14) corresponding to the shortest path is globally asymptotically stable:

1. If (3.19) holds, then $A_{1}$ is globally asymptotically stable.

2. If (3.20) holds, then $A_{2}$ is globally asymptotically stable. 
3. If (3.22) holds, then $A_{3}$ is globally asymptotically stable.

4. If (3.23) holds, then $A_{4}$ is globally asymptotically stable.

Acknowledgement The second author deeply appreciate Professor Ryo Kobayashi in Hiroshima University, who lead him to mathematical analysis of this problem about two years ago in a small seminar room in Research Institute for Electronic Science in Hokkaido University. He is now very glad to answer partially some of the questions asked at that time. Moreover, the authors make a sincere expression of appreciation to the referee for his careful proof-reading of the first manuscript and his helpful advices. The first author is supported by Grant of No. b035 of the program: "Initiative program of facinating educations of graduate school" in Ministry of Education, Culture, Sports, Science and Technology in Japan, and the second author is supported by Grant No. 17540118 of JSPS.

\section{References}

[1] Fan M. and Zou X., Global asymptotic stability of a class nonautonomous integrodifferential systems and applications. Nonlinear Analysis 57 (2004), 111-135.

[2] LaSalle J.P. and Lefschetz S., Stability by Liapunov's Direct Method. Academic Press, New York, 1961.

[3] Matano H., Iwanami course Applied mathematics 4 Differential Equation I. (in Japanese), Iwanami Shoten, 1993.

[4] Nakagaki T., Yamada H. and Hara H., Smart network solution by an amoeboid organism. Biophys. Chem. 107 (2004), 1-5.

[5] Nakagaki T., Yamada H. and Tóth Á., Maze-solving by an amoeboid organism. Nature 407 (2000), 470.

[6] Nakagaki T., Yamada H. and Tóth Á., Path finding by tube morphogenesis in an amoeboid organism. Biophys. Chem. 92 (2001), 47-52.

[7] Tero A., Kobayashi R. and Nakagaki T., A coupled-oscillator model with a conservation law for the rhythmic amoeboid movements of plasmodial slime molds. Physica D 205 (2005), 125-135.

[ 8 ] Tero A., Kobayashi R. and Nakagaki T., A Mathematical model for adaptive transport network in path finding by true slime mold. to appear in J. theor. Biol.

[9] Tero A., Kobayashi R. and Nakagaki T., Physarum solver:-a biologically inspired method of road-network navigation- ${ }^{-}$to appear in Physica A. 
T. Miyaji

Department of Mathematical and Life Sciences Graduate School of Science

Hiroshima University

1-3-1 Kagamiyama

Higashi-Hiroshima 739-8526 Japan

I. Ohnishi

Department of Mathematical and Life Sciences Graduate School of Science

Hiroshima University

1-3-1 Kagamiyama

Higashi-Hiroshima 739-8526 Japan 